\title{
Hepatitis B Virus-Related Nephropathy and Lupus Nephritis: Morphologic Similarities of Two Clinical Entities
}

\author{
F. Mac-Moune Lai, M.D., K.F. To, M.B.Ch.B., A.Y.M. Wang, M.B.B.S., P.C.L. Choi, M.B.Ch.B., \\ C.C. Szeto, M.B.Ch.B., P.K.T. Li, M.B.B.S., C.B. Leung, M.B.Ch.B., K.N. Lai, M.D. \\ Departments of Anatomical \& Cellular Pathology (FM-ML, KFT, PCLC) and Medicine \& Therapeutics \\ (AYMW, CCS, PKTL, CBL, KNL), The Chinese University of Hong Kong, Shatin, Hong Kong
}

We compared the clinicopathologic features of 22 patients with hepatitis $B$ virus-related membranous nephropathy, all with detectable glomerular hepatitis $B$ e antigen, and of 26 patients with lupus nephritis class $V$. Both groups of patients similarly presented with heavy proteinuria or nephrotic syndrome; however, the patients with hepatitis B virusrelated membranous nephropathy, who were predominantly male, did not have the extrarenal manifestations and autoantibodies seen in systemic lupus erythematosus. The glomerular lesions in both clinical entities were similar and at times indistinguishable, demonstrating polyclonal immunoglobulins and polytypic complements in similar subepithelial ultrastructural distribution. No morphologic feature, single or combined, carrying a high positive predictive value for the diagnosis of either nephritis was identified. Lesions such as hematoxyphil bodies and fingerprint dense deposits, distinctive of systemic lupus erythematosus, were rarely found. At the time of biopsy, when systemic lupus erythematosus is not clinically suspected, the diagnosis between hepatitis $B$ virus-related membranous nephropathy and lupus nephritis may be difficult or impossible to differentiate, especially in geographic areas where both lupus nephritis and hepatitis B surface antigen carriers are common. This study focused on the use of specific monoclonal antisera to detect glomerular hepatitis $B$ virus antigens, which contribute to the diagnosis of hepatitis $B$ virus-related nephritis.

KEY WORDS: Antinuclear antibodies, Hematoxyphil bodies, Hepatitis B virus, Hepatitis B virus-

Copyright () 2000 by The United States and Canadian Academy of Pathology, Inc.

VOL. 13, NO. 2, P. 166, 2000 Printed in the U.S.A.

Date of acceptance: August 24, 1999.

Address reprint requests to: Dr. Fernand Mac-Moune Lai, Department of

Anatomical \& Cellular Pathology, Prince of Wales Hospital, Room 34055

Shatin, Hong Kong; e-mail: fmlai@cuhk.edu.hk; fax: 852-2637-6274. related nephropathy, Lupus nephritis, Membranous nephropathy, Systemic lupus erythematosus.

Mod Pathol 2000;13(2):166-172

The implication of the hepatitis B virus (HBV) in glomerulonephritis is widely recognized (1-4). The glomerular lesions, which appear pathogenetically linked to the virus and demonstrate presence of viral antigens, include membranous nephropathy, mesangiocapillary glomerulonephritis (membranoproliferative glomerulonephritis [MPGN]), a subset of IgA nephropathy, and the morphologic overlaps that these nephritides may assume $(1,4-8)$. Such a definition of HBV-related glomerulonephritis (HBVGN) arguably may seem restrictive, but these glomerular lesions were reproducible in experimental animals and resolved after HBV seroconversion in humans $(2,5,9)$. HBVGN is also characterized by immune depositions of polyclonal immunoglobulins associated with polytypic complements in glomeruli, features that are reminiscent of lupus nephritis $(6,8,10)$.

In Hong Kong, where $10 \%$ of the population carry the hepatitis B surface antigen (HBsAg) and where lupus nephritis is also common, we are often confronted with the diagnostic dilemma of differentiating HBVGN from lupus nephritis, particularly when membranous nephropathy is the predominant lesion and when at the time of biopsy the diagnosis of systemic lupus erythematosus (SLE) is uncertain and serologies are unavailable $(2,6,11$, 12). Even when the serology is known, a mere positive serum HBsAg does not necessarily link HBV with the underlying glomerulonephritis, as nearly half of the seropositive patients with primary glomerulopathy do not demonstrate glomerular viral antigens $(6,13)$. From both pathogenetic and clinical viewpoints, the study of glomerular viral antigens is important for the diagnosis of HBVGN (6). The diagnostic problem between HBVGN and lupus nephritis may be further compounded in patients 
who have SLE and a positive serum HBsAg, as it may be uncertain whether these patients have lupus nephritis or HBVGN or both $(6,11)$. This study did not address this issue but focused on the comparison of HBV-related membranous nephropathy (HBVMN) and lupus nephritis class V (LMN) to identify clinicopathologic features that allow their distinction.

\section{MATERIALS AND METHODS}

\section{Patients}

The cases were retrieved from our files from 1986 to 1994 for all patients with the diagnosis of membranous nephropathy (MGN). Of 40 patients with both a positive serum HBsAg and MGN, pure or mixed with mesangiocapillary glomerulonephritis (MCGN), 22 were selected by the following criteria: (1) absence of systemic disease (two patients with diabetes and three patients with lupus were excluded), (2) presence of a positive HBsAg serology before and 3 months after biopsy (one patient was excluded for seroconversion), and (3) presence of detectable glomerular hepatitis $\mathrm{B}$ e antigen (HBeAg). Also excluded were three repeated biopsies, five cases with insufficient materials, and six cases with no demonstrable HBeAg. Twenty-six patients with lupus nephritis were selected on the basis of the following: (1) clinical diagnosis of SLE with at least four American Rheumatic Association criteria; (2) negative serum HBsAg; and (3) diagnosis of LMN, which may show mesangial hypercellularity and interposition into capillary basement membrane (mixed MGN-MCGN). Patients who had previously been treated with cytotoxic drugs or methylprednisolone were excluded. Sixteen patients with HBVMN and 20 with LMN had MGN, respectively; 6 cases in each group had mixed MGN-MCGN.

\section{Clinical Features and Serology}

In both groups, the features that were compared included age, sex, proteinuria, nephrotic syndrome, arthralgia, skin rash, oral ulcer, cytopenia, serositis, quantitative proteinuria, serum creatinine, glomerular filtration rate, and serum C3 and C4 levels. Serum HBsAg, HBeAg, and anti-HBs were profiled at the time of renal biopsy and at least twice 3 to 12 months after by standard immunoassay methods previously described $(3,6,13-15)$. Serum for antinuclear autoantibodies (ANA) and anti-dsDNA was also compared.

\section{Renal Biopsies}

Light microscopy examined mesangial hypercellularity, cellular crescent, mesangial sclerosis, tubular atrophy, interstitial inflammatory and foam cells, hyaline arteriolosclerosis, wire-loop, hyaline thrombi, necrotizing lesions, and hematoxyphil bodies.

In immunofluorescence study, fluorescein isothiocyanate, conjugated-labeled $\mathrm{F}\left(\mathrm{ab}^{\prime}\right) 2$ fragments of murine monoclonal antibodies were used to examine HBsAg, HBcAg, and HBeAg in frozen sections, and their specificity and properties were previously reported $(3,6,11,13)$. All HBVMN and only eight cases of LMN were examined. Control immunostainings included 6 non-HBsAg carriers with primary MGN, 10 postmortem kidneys from HBsAg carriers with absence of renal disease, and 8 kidneys from non-HBsAg carriers who died of traumatic injury $(6,11,13)$. Polyclonal antibodies against IgA, IgG, IgM, C1q, C3, C4, and fibrinogen were used, all at the 1:100 dilution $(6,11,13)$. The immunostaining was scored on a scale of 0 to $4+$ without prior knowledge of the nature of antibody used. Electron microscopy examined two glomeruli in each case for epithelial foot process fusion, electron dense depositions and their distribution, virallike particles, tubuloreticular structures, and fingerprint immune deposits.

\section{RESULTS}

The statistical analysis in both groups was assessed as two independent samples using the Mann-Whitney U nonparametric variable with the SSCP software (Tables 1-3).

All 26 patients with SLE met at any one time at least four diagnostic criteria, including arthralgia, skin rash, oral ulcer, cytopenia, pleuritis, ANA or anti-dsDNA, and nephritis. At the time of biopsy, 16 patients were known to have SLE, 4 had a suggestive diagnosis, and 6 were not suspected to have

TABLE 1. Light Microscopic Features Contrasting HBVMN and LMN

\begin{tabular}{lccccccccccccc}
\hline \multicolumn{1}{c}{ Group } & MC & EC & Sc & TA & IFC & IIC & HTN & WLL & H \\
\hline HBVMN (\%) $(n=22)$ & $16(72.7)$ & $2(9.1)$ & $8(36.4)$ & $6(27.2)$ & $10(45.5)$ & $5(22.7)$ & $3(13.6)$ & $6(27.3)$ & $2(9.1)$ & $2(9.1)$ & $0(0)$ & \\
LMN (\%) $(n=26)$ & $23(88.5)$ & $9(34.6)$ & $9(34.6)$ & $14(43.8)$ & $3(11.5)$ & $16(61.5)$ & $4(15.4)$ & $11(42.3)$ & $12(46.2)$ & $7(27.0)$ & $2(7.7)$ \\
$p$ & 0.01 & 0.02 & ns & ns & 0.007 & 0.004 & ns & ns & 0.005 & ns & ns \\
\hline
\end{tabular}

HBVMN, hepatitis B virus-related membranous nephropathy; LMN, lupus nephritis class V; MC, mesangial hypercellularity, more than 3 cells per area; EC, epithelial crescent; Sc, sclerosis, expansion of mesangial matrix, with or without collapse of tufts; TA, tubular atrophy; IFC, interstitial foam cells; IIC, interstitial inflammatory cells; HTN, hypertensive arteriolosclerosis; WLL, wire-loop like lesion; N, necrotizing lesion (disrupted glomerular capillaries, polymorphs, fibrin deposits); T, hyaline thrombin; HB, hematoxyphil bodies. 
TABLE 2. Glomerular Immunofluorescence Results of HBVMN and LMN

\begin{tabular}{|c|c|c|c|c|c|c|c|c|c|}
\hline Group & HBsAg & HBeAg & A & G & M & $\mathrm{Clq}$ & C3 & $\mathrm{C} 4$ & F \\
\hline HBVMN $(\%)(n=22)$ & $8(35.4)$ & $22(100.0)$ & $16(72.7)$ & $22(100.0)$ & $16(72.7)$ & $16(72.7)$ & $22(100.0)$ & $12(54.6)$ & $4(18.2)$ \\
\hline LMN (\%) $(n=26)$ & $0(0)$ & $0(0)$ & $26(100.0)$ & $26(100.0)$ & $19(73.0)$ & $25(96.0)$ & $26(100.0)$ & $18(69.2)$ & $15(57.7)$ \\
\hline$p$ & 0.0008 & 0.0001 & 0.001 & ns & ns & 0.002 & ns & ns & 0.002 \\
\hline
\end{tabular}

HBVMN, hepatitis B virus-related membranous nephropathy; LMN, lupus nephritis class V; HBsAg, hepatitis B surface antigen; HBeAg, hepatitis B e antigen; C3 and C4, serum C3 and C4 in mg/L; F, fibrinogen in capillary tufts.

TABLE 3. Electron Microscopic Features in HBVMN and LMN

\begin{tabular}{|c|c|c|c|c|c|c|c|}
\hline Group & FPF & Subepithelial EDD & Subendothelial EDD & Mesangial EDD & VLP & TRS & FP \\
\hline HBVMN (\%) $(n=22)$ & $18(81.8)$ & $22(100.0)$ & $15(68.2)$ & $11(50.0)$ & $4(18.2)$ & $3(13.4)$ & $0(0)$ \\
\hline $\operatorname{LMN}(\%)(n=26)$ & 25 (96.2) & $26(100.0)$ & $21(80.8)$ & $20(76.9)$ & $5(19.2)$ & $19(73.4)$ & $2(7.7)$ \\
\hline$p$ & ns & ns & ns & ns & ns & 0.001 & ns \\
\hline
\end{tabular}

HBVMN, hepatitis B virus-related membranous nephropathy; LMN, lupus nephritis class V; FPF, fusion of epithelial foot processes; EDD, electron dense deposits; VLP, viral-like particles; TRS, tubuloreticular structures; FP, fingerprint immune deposits.

TABLE 4. Clinical Features Contrasting Patients with HBVMN and LMN

\begin{tabular}{|c|c|c|c|c|c|c|c|c|c|c|c|}
\hline Group & Sex $(M: F)$ & Age & Prot & $\mathrm{SCr}$ & GFR & Salb & C3 & $\mathrm{C} 4$ & ESR & ANA & Anti-dsDNA \\
\hline HBVMN $(n=22)$ & $18: 4$ & 30.0 & 3.42 & 95.6 & 89.2 & 27.0 & 970 & 229 & 53 & $10 \%$ & $0 \%$ \\
\hline LMN $(n=26)$ & $3: 23$ & 30.2 & 4.79 & 113.0 & 85.1 & 25.8 & 511 & 231 & 92 & $92 \%$ & $58 \%$ \\
\hline$p$ & $<.0001$ & ns & ns & ns & ns & ns & 0.0015 & ns & ns & $<.0001$ & $<.0001$ \\
\hline
\end{tabular}

HBVMN, hepatitis B virus-related membranous nephropathy; LMN, lupus nephritis class V; Prot, proteinuria in g/24h; SCr, serum creatinine in $\mu \mathrm{mol} / \mathrm{L}$; GFR, glomerular filtration rate in ml/min; Salb, serum albumin in g/L; C3 and C4, serum C3 \& C4 in mg/L; ESR, erythrocyte sedimentation rate; ns, statistically not significant.

SLE. None of the 22 patients with HBVMN fulfilled the criteria for SLE, and only 5 of them had arthralgia and/or cytopenia but not the other symptoms. Table 4 depicts additional clinical features in both groups. Eighty-two percent of patients with HBVMN were male, with a mean age of 30 years, proteinuria of $3.42 \mathrm{~g}$, and serum creatinine of 1.08 $\mathrm{mg} / \mathrm{dL}$. They presented with nephrotic syndrome (50\%), proteinuria $(32 \%)$, or edema (18\%). Most of the patients with LMN were female (89\%), with a mean age of 30.2 years, proteinuria of $4.79 \mathrm{~g}$, and serum creatinine of $1.26 \mathrm{mg} / \mathrm{dL}$. They also presented with nephrotic syndrome $(42 \%)$, proteinuria $(27 \%)$, or edema $(8 \%)$. By definition, all patients with HBVMN had a positive serum HBsAg, and 17 also tested positive for HBeAg. All patients with LMN had negative serum HBsAg and HBeAg in 14 cases tested. Only 2 of 19 patients with HBVMN had a positive ANA, with a low titer of 1:80, and none of the 11 patients tested had anti-dsDNA. ANA equal to or more than 1:80 was found in 24 of 26 patients with SLE, and 11 of 19 tested had anti-dsDNA.

The light microscopic findings are shown in Table 1. In both groups, thickened capillary walls demonstrated "spikes" and "honeycomb" patterns in silver stain (Fig. 1). The hyaline capillary thickening was sometimes reminiscent of "wire-loop" lesion; however, on electron microscopy, these hyaline changes were due to heavy subepithelial deposits, as subendothelial immune deposits were absent or scanty. Mesangial hypercellularity was mild to moderate, with four to six cells per area, and was significantly more pronounced in LMN. In mixed

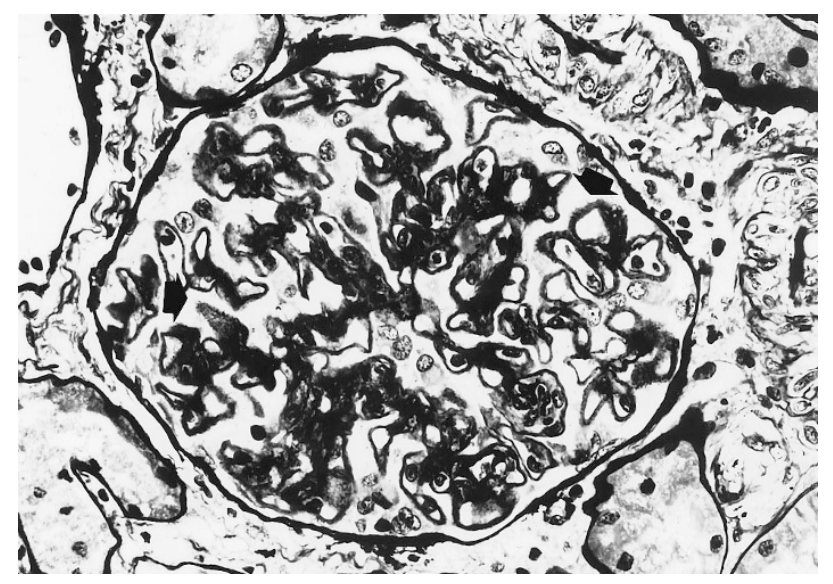

FIGURE 1. Lupus nephritis class V, characterized by the presence of "spikes" and "honeycomb" lesions (arrows) (periodic acid-silver methenamine, $360 \times)$.

MGN-MCGN, cells' interposition led to duplication and "tram-track" appearance of capillary walls (Fig. 2). Cellular crescents were present in $9.1 \%$ of HBVGN, but they were significantly more common in LMN (38.5\%) (Fig. 3). These crescents were focal and usually segmental and seemed to be correlated with necrosis seen in $9.1 \%$ and $46.2 \%$, respectively. Mesangial sclerosis, with or without segmental collapse tufts, affected approximately one third of the cases in each group. Tubular atrophy was similar in both nephritides. Interstitial foam cells were pronounced in HBVMN (45.5\%), but mononuclear cells were more common in LMN (61.5\%). Hyaline arteriolosclerosis was rare. Although hematoxyphil 


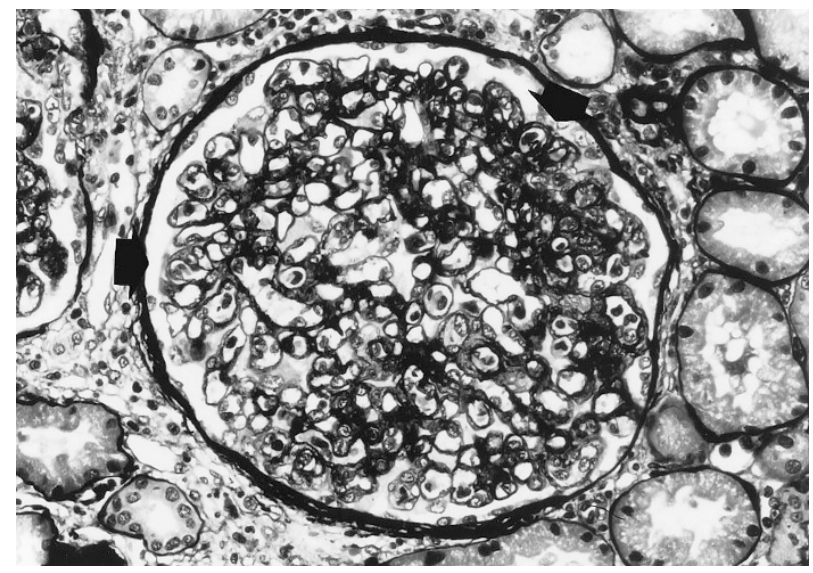

FIGURE 2. Hepatitis B virus-related membranous nephropathy mixed with mesangiocapillary glomerulonephritis demonstrating duplication of capillary wall with a "tram-track" appearance (arrows) (periodic acid-silver methenamine, $360 \times$ ).

bodies were distinctive in SLE, they were seen only in two patients, but not in patients with HBVGN.

Results of immunofluorescence studies are shown in Table 2. As selected, all HBVMN had detectable HBeAg in glomeruli (Fig. 4), and eight of them also showed HBsAg. None of the LMN, the non-HBsAg controls, or the postmortem kidneys demonstrated HBV antigens. Both nephritides exhibited a "full house" immunofluorescence pattern and showed a high prevalence of polyclonal immunoglobulins and polytypic complement from 54.6 to $100 \%$ of cases, although IgA, C1q, and fibrin were more frequent in LMN. The intensity was strong for IgG, Clq, and C3; intermediate for IgA and IgM; but similar in both groups. Tubular basement membrane staining was noted in three patients with SLE but in no patients with HBVGN.

All cases were characterized by diffuse subepithelial electron dense deposits and fusion of epithelial foot processes (Fig. 5). Subendothelial and mesangial deposits were common but were usually small and scattered, unlike the broad, heavy subendothelial complexes seen in proliferative lupus nephritis (10). A few patients in each group revealed clusters of viral-like particles within immune deposits (Fig. 6). Distinctive lesions, such as tubuloreticular structures, were present in $73.1 \%$ of LMN but were also detected in three cases of HBVMN (Fig. 7), and fingerprint in dense deposits were recognized in two cases of LMN but not in HBVMN (Fig. 8).

\section{DISCUSSION}

This study was initiated because we often have been confronted with the diagnostic problem of MGN associated with polyclonal and polytypic complement immune complexes. Although such a full-house immunoreactant glomerular pattern generally implies a diagnosis of lupus nephritis, in

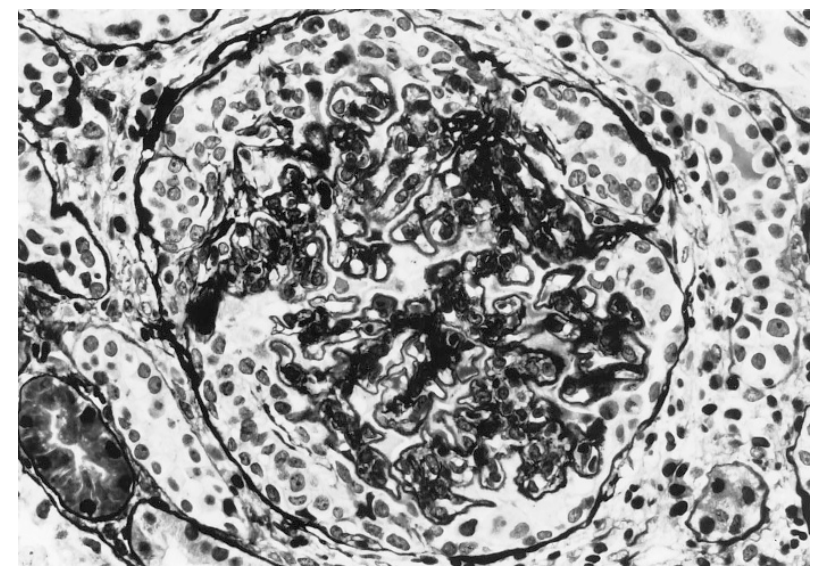

FIGURE 3. Hepatitis B virus-related nephropathy with cellular crescent is infrequent, but such a lesion is more common in lupus nephritis class $\mathrm{V}$ (periodic acid-silver methenamine, $380 \times$ ).

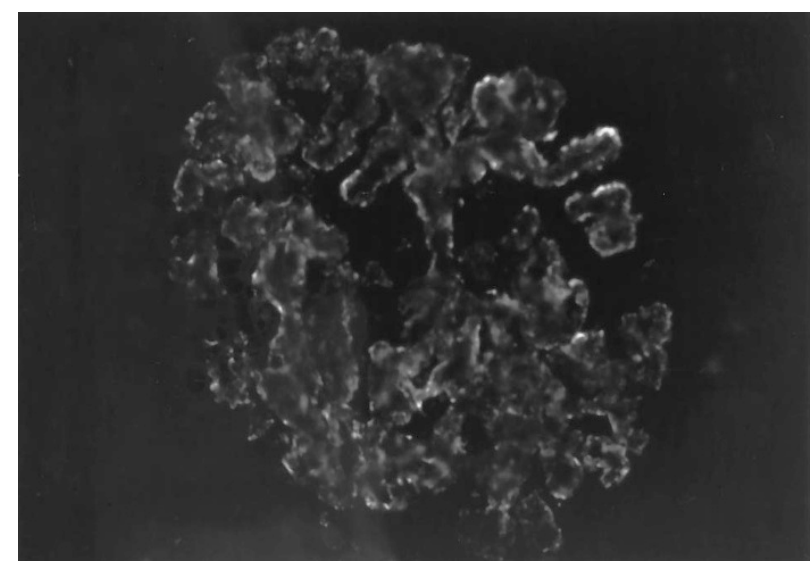

FIGURE 4. Immunofluorescence detection of glomerular HBeAg, distributed along the glomerular capillary wall (monoclonal F[ab']2 anti-hepatitis B e antibody, $360 \times$ ).

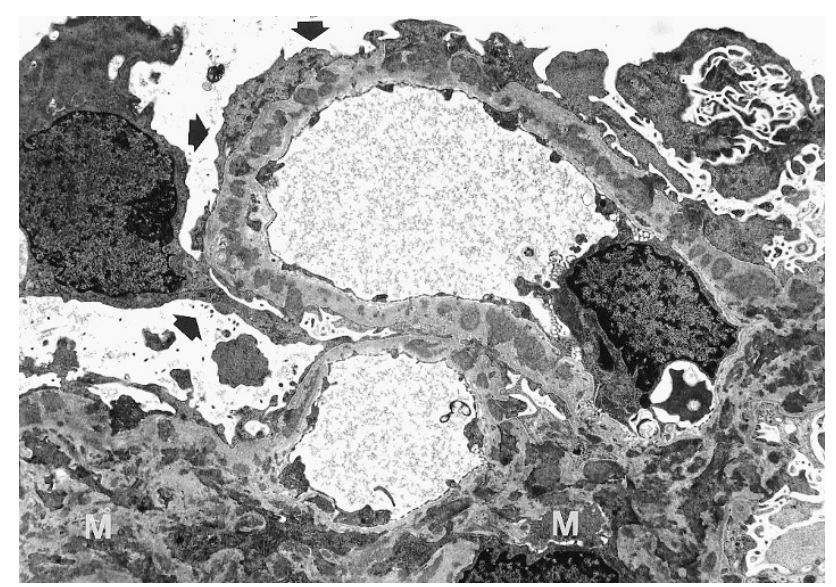

FIGURE 5. Both nephritides typically show subepithelial and mesangial electron dense deposits (M) and fusion of epithelial foot processes (arrows) (lead citrate and uranyl acetate, $5800 \times$ ).

Hong Kong, where HBV infection is endemic, HBVGN is an alternative possibility $(4,6,8)$. Such a polyclonal pattern in HBVGN has also previously been reported in nonendemic areas (12). The diag- 


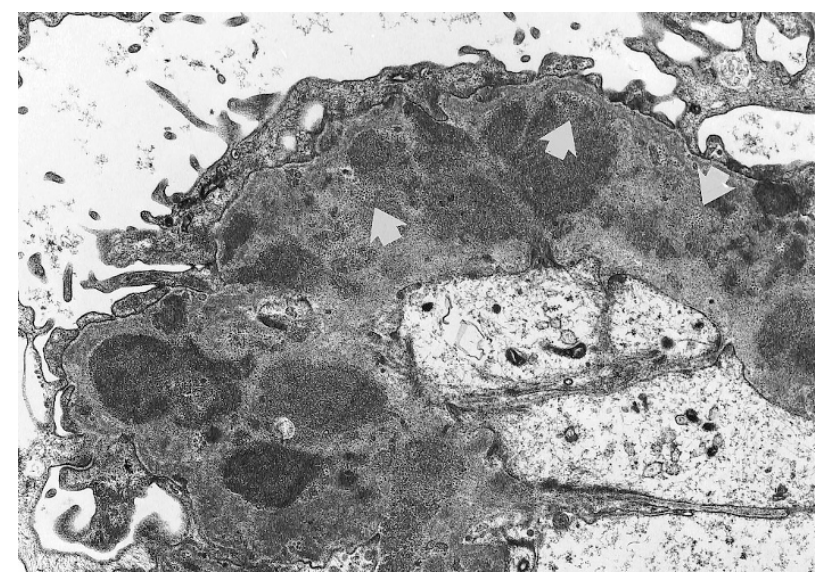

FIGURE 6. Viral-like particles (arrows) associated with electron dense deposits were detected in both conditions (lead citrate and uranyl acetate, $13,000 \times$ ).

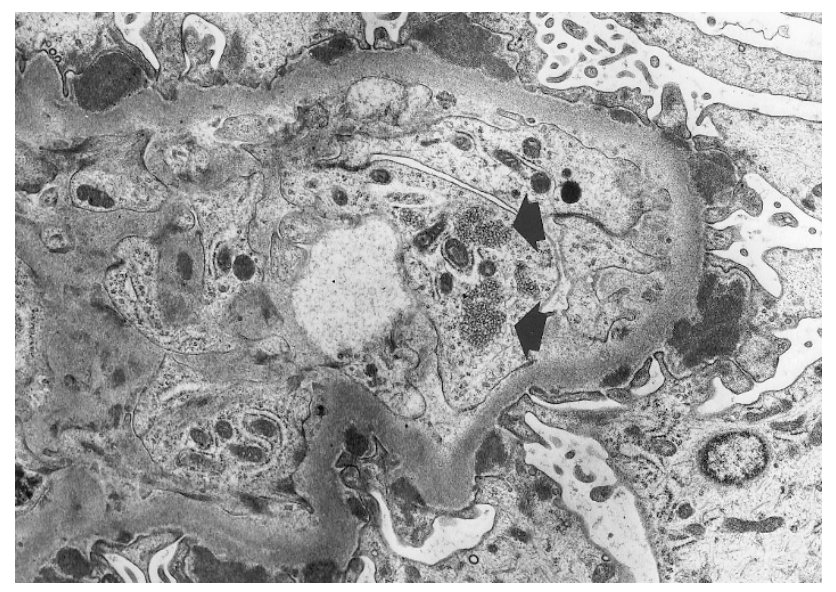

FIGURE 7. Tubuloreticular structures (arrows), seen in both nephritides, carries the highest predictive value in the diagnosis of lupus nephritis (lead citrate and uranyl acetate, $7000 \times$ ).

nosis of lupus nephritis poses no problem when this full-house pattern accompanies a diffuse proliferative glomerulonephritis, because such a lesion is rarely, if ever, a form of $\operatorname{HBVGN}(4,6,8)$. The differential diagnosis between HBVMN and LMN is challenging at the time of biopsy, when clinical features of SLE are not suspected or are lacking (10, 16). This problem is compounded further in patients with SLE who also carry HBsAg, as they may develop HBVGN or a nephropathy other than lupus nephritis or that may superimpose with lupus nephritis $(11,17)$. This study did not address these cases but rather aimed to identify clinical or pathologic features that may differentiate these two nephritides. Thus, in patient selection, it was important to include only cases with demonstrable HBeAg in glomeruli to optimize representation of the HBVMN group and to exclude patients with SLE and a positive HBsAg serum. One might argue that presence of glomerular HBV antigens does not establish with absolute certainty the link between HBV infection and MGN; however, such a causal

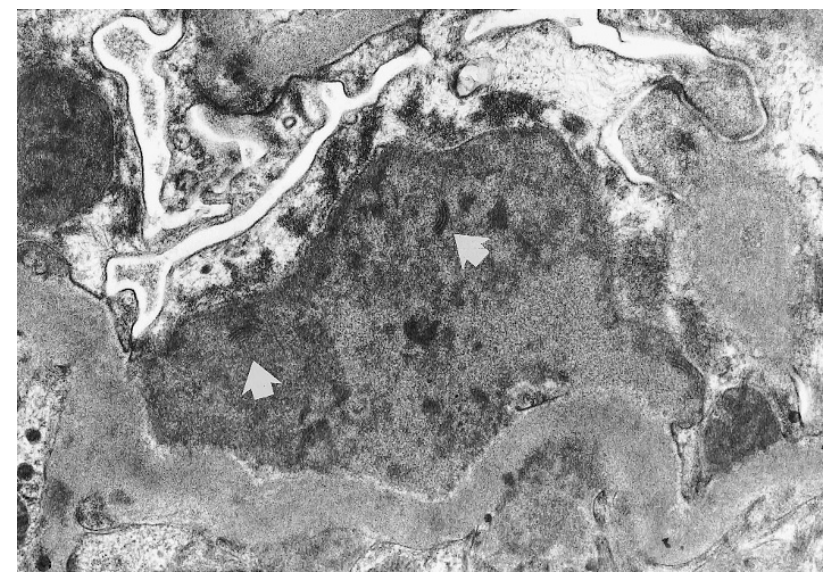

FIGURE 8. Fingerprint immune dense deposits (arrows) are distinctive of lupus nephritis, but they are uncommonly seen in lupus nephritis class V (lead citrate and uranyl acetate, 14,000×).

relationship has been reproduced in animal models and supported by the resolution of MGN in patients with HBV seroconversion $(2,5,9)$. Perhaps it is more arguable from a clinical viewpoint to classify these MGN as idiopathic and dismiss the significance of the positive HBsAg serology and the detectable viral antigens in glomeruli (1, 3-6).

Although HBV infection may present with a serum sickness-like syndrome, symptoms are uncommon in those who chronically carry HBsAg, and only a few of our patients with HBVMN showed extrarenal symptoms. None of them met more than three clinical criteria, including nephritis, to fulfill the diagnosis of SLE $(2,6,10)$. Therefore, only patients with SLE and insufficient criteria or limited clinical manifestations at the time of renal biopsy can cause diagnostic confusion with HBVGN (10, $16,17)$. The minimum of four clinical criteria, a high titer of ANA, and a positive anti-dsDNA serology all remain important to the diagnosis of lupus nephritis, with perhaps the exception of patients with SLE who also carry HBsAg, and such patients were excluded from this study $(6,10,11,17)$.

The female predominance in SLE and lupus nephritis is widely known and has been attributed to genetic and hormonal factors, although no specific genetic markers have so far been recognized $(10,18$, 19). This series reveals a strong predilection of HBVMN in males and marks a striking contrast with that in lupus nephritis, but such difference has only limited diagnostic value at the individual level. The strong male predilection in HBVMN cannot be explained by the slightly higher HBsAg carrier rate of $10 \%$ in the male population, compared with $8 \%$ in women, but may suggest the influence exerted by genetic or hormonal factors $(2,7,19)$. As expected, the renal manifestations in both groups, including level of proteinuria, serum creatinine, and glomerular filtration rate, were similar. The significantly lower serum C3 level in patients with SLE may 
reflect a more extensive extrarenal disease in these patients, although a low serum complement in HBV-related nephritis has also previously been recognized $(10,12)$.

The scope of this study was limited to pure MGN or mixed MGN-MCGN as they constitute the most widely recognized lesions in $\operatorname{HBVGN}(4,6)$. Despite this morphologic restriction, we attempted to identify differences that may be distinctive between the two conditions. Although significant differences were observed in mesangial hypercellularity, cellular crescent, interstitial foam cells and inflammatory cells, and necrosis, none of these features is specific to establish a diagnosis. When these features were analyzed by logistic regression, interstitial inflammatory cells had the most predictive value in light microscopy to determine lupus nephritis, but such interstitial infiltrates were not distinctive and were also seen in HBVMN. In SLE, interstitial infiltrates have been attributed to cellmediated immunity and shown to contribute to the activity and progression of the nephritis $(18,20)$. Hematoxyphil bodies and antibody-bound nuclear debris were rarely seen in LMN but remain distinctive of the diagnosis in SLE, as they were absent in HBVMN (8).

In both conditions, the characteristic polyclonal immunoglobulins and polytypic complement deposits and the overall high prevalence of immunoreactants minimize the significance in their different IgA and Clq immunoreactivity. These immunoreactants routinely used in diagnosis in renal biopsy do not permit a distinction between LMN and HBVMN. Although the absence of glomerular HBV antigens in LMN is expected, this also excludes antibody cross-reactivity with the heterogeneous immune complexes in this nephritis. This observation stresses the importance of using specific monoclonal $\mathrm{F}\left(\mathrm{ab}^{\prime}\right) 2$ antibody fragments and enhances the significance of detectable glomerular HBV antigens in HBVMN (6). In fact, we advocate using the presence of glomerular $\mathrm{HBV}$ antigens as a diagnostic criterion in HBVGN $(1-3,6)$. These specific viral antisera have permitted recognition of HBV-related nephropathy, including in patients with SLE or other systemic diseases $(6,11)$. Similar "etiologic" antisera can be developed in other glomerulopathies to identify specific causes or pathogenesis and to go beyond the simple morphologic label. Molecular probes that trace HBV genomic "footprints" in kidney are useful, but their role in diagnosis and pathogenesis of nephritis needs to be assessed (21). Apart from fingerprint immune deposits, which were rarely seen, no ultrastructure permitted distinction between the two nephritides. Although tubuloreticular structures were seen in both conditions, they were common in LMN and they carry from logistic regression analysis of all morphologic features the highest predictive diagnostic value for SLE.

Although the pathogenesis and pathophysiology of HBVGN and LMN are not completely understood, their close morphologic similarities, especially the similar full-house immunofluorescence pattern, seem to set them apart from other types of membranous nephropathy, such as gold-induced or tumor-associated examples, and revive the notion that a latent viral infection may be implicated in the development of SLE. The search for a virus, however, has remained unfruitful. The viral-like particles seen here lack the features of a complete virion, although they are found in receding immune complexes possibly induced by virus (6). In contrast to HBVGN, in which viral replication seems to be contemporary to glomerular injury, SLE is thought to result from faulty autoimmunity initiated by a virus, which has subsequently vanished $(10,21)$.

This study concludes that HBVMN and LMN can be morphologically indistinct, with no single or combined features carrying a positive predictive value for their individual diagnosis. Interstitial infiltrates and tubuloreticular structures, which carry a relative and highest predictive value, both are nonspecific of SLE. Although clinical manifestations and criteria may help to distinguish the two conditions and to establish the diagnosis of lupus nephritis, a positive HBsAg serology in patients with SLE would offset such a conclusion. In any event, the detection of viral antigens in glomeruli by the use of specific monoclonal antisera may permit us to link the virus with the glomerular lesion, as in both the human and experimental models $(1,5,6)$.

\section{REFERENCES}

1. Combes B, Stastny P, Shorey J, Eigenbrodt EH, Barrera A, Hull AR, et al. Glomerulonephritis with deposition of Australia antigen-antibody complexes in glomerular basement membrane. Lancet 1971;2:234-7.

2. Lai KN, Li PKT, Lui SF, Au TC, Tam JSL, Tong KL, et al. Membranous nephropathy related to hepatitis B virus in adults. N Engl J Med 1991;324:1457-63.

3. Hirose H, Udo K, Kojima M, Takahashi Y, Miyakawa Y, Miyamoto K, et al. Deposition of hepatitis B e antigen in membranous glomerulonephritis: identification by $\mathrm{F}\left(\mathrm{ab}^{\prime}\right) 2$ fragments of monoclonal antibody. Kidney Int 1984;26:33841.

4. Venkataseshan VS, Lieberman K, Kim DU, Thung SN, Dikman S, D'Agati V, et al. Hepatitis-B-associated glomerulonephritis: pathology, pathogenesis, and clinical course. Medicine 1990;69:200-16.

5. Peters DN, Steinberg H, Anderson WI, Hornbuckle WE, Cote PJ, Gerin JL, et al. Immunopathology of glomerulonephritis associated with chronic woodchuck hepatitis virus infection in woodchucks (Marmota monax). Am J Pathol 1992;141: 143-52.

6. Mac-Moune Lai F, Lai KN, Tam JS, Lui SF, To KF, Li PKT. Primary glomerulonephritis with detectable glomerular hep- 
atitis B virus antigen. Am J Surg Pathol 1994;18:175-86.

7. Johnson RJ, Couser WG. Hepatitis B infection and renal disease: clinical, immunopathogenetic and therapeutic considerations. Kidney Int 1990;37:663-76.

8. Churg J, Bernstein J, Glassock RJ. Glomerulonephritis of systemic diseases. In: Churg J, Bernstein J, Glassock RJ, editors. Renal disease: classification and atlas of glomerular diseases. Tokyo: Igaku-Shoin; 1995. pp. 151-79.

9. Schneeberger EE, Leber PD, Karnovsky MJ, McCluskey RT. Altered properties of the renal glomerulus in autologous immune complex nephritis: an ultrastructural tracer study. J Exp Med 1974;139:1283-302.

10. Tan EM, Cohen AS, Fries JF, Masi AT, McShane DJ, Rothfield $\mathrm{NF}$, et al. The revised criteria for the classification of systemic lupus erythematosus. Arthritis Rheum 1982;25:1271-7.

11. Mac-Moune Lai F, Lai KN, Lee JCK, Hom BL. Hepatitis B virus-related glomerulopathy in patients with systemic lupus erythematosus. Am J Clin Pathol 1987;88:412-20.

12. Southwest Pediatric Nephrology Study Group. Hepatitis B surface antigenemia in North American children with membranous glomerulonephropathy. J Pediatr 1985;106:571-8.

13. Lai KN, Mac-Moune Lai F, Tam JS. Comparison of polyclonal and monoclonal antibodies in determination of glomerular deposits of hepatitis B virus antigens in hepatitis B virusassociated glomerulonephritides. Am J Clin Pathol 1989;92: $159-65$.
14. Lai KN, Mac-Moune Lai F, Tam JS. IgA nephropathy associated with chronic hepatitis B virus infection in adults: the pathogenetic role of HBsAg. J Pathol 1989;157:321-7.

15. Mac-Moune Lai F, Tam JS, Li PK, Lai KN. Replication of hepatitis B virus with corticosteroid therapy in hepatitis B virus related membranous nephropathy. Virchows Arch A Pathol Anat Histopathol 1989;414:279-84.

16. Cavallo T, Cameron WR, Lapenas D. Immunopathology of early and clinically silent lupus nephropathy. Am J Pathol 1977;87:1-18.

17. Hebert LA, Sharma HM, Sedmak DD, Bay WH. Unexpected renal biopsy findings in a febrile systemic lupus erythematosus patient with worsening of renal function and heavy proteinuria. Am J Kidney Dis 1989;13:504-7.

18. Steinberg AD. Systemic lupus erythematosus: theories of pathogenesis and approach to therapy. Clin Immunol Immunopathol 1994;72:171-6.

19. Sequeira JF, Keser G, Greenstein B, Wheeler MJ, Duarte PC, Khamashata MA, et al. Systemic lupus erythematosus: sex hormones in male patients. Lupus 1993;2:315-7.

20. Alexopoulos E, Seron D, Hartley RB, Cameron JS. Lupus nephritis: correlation of interstitial cells with glomerular function. Kidney Int 1990;37:100-9.

21. Lai KN, Ho RTH, Tam JS, Mac-Moune Lai F. Detection of hepatitis B virus DNA and RNA in kidneys of HBV-related glomerulonephritis. Kidney Int 1996;50:1965-77. 